Korean J Ophthalmol 2021;35(3):244-245 https://doi.org/10.3341/kjo.2020.0111

\section{Isolated Unilateral Tessier Cleft 10 with Anterior Staphyloma}

\section{Dear Editor,}

Tessier developmental cleft anomalies are exceedingly rare situations. The nature and extent of the anomaly varies according to the cleft number. Cleft number 10 is the most uncommon of them all. Herein the authors report a similar situation wherein child was diagnosed as a case of Tessier cleft 10 with anterior staphyloma.

A 9-month-old male child born out of nonconsanguineous marriage was brought with a history of abnormal right eye since birth (Fig. 1A). General examination did not reveal any obvious systemic abnormality. On ophthalmological examination, the child was able to fix and follow the light with his left eye but resisted occlusion of the same, indicating poor vision in the right eye. The right side demonstrated progressive protrusion of the right eye bulb with thinning of the sclera perilimbal and on nasal end denoting anterior staphyloma with a disorganized globe; the upper eye lid had three components: the lateral and medial components which were vertically aligned, with the presence of cilia and a middle colobomatous part which occupied more than half of the eyelid and extended across the eyebrow into the forehead ending with a notch (Fig. 1B). Anterior segment and fundus examination were within normal limits on the left side.

Tessier introduced facial cleft classification in 1976, since then this nomenclature has gained a lot of popularity and worldwide acceptance. The classification specifies numbers ranging from 0 to 14 , to multiple axes passing through eyebrows, eyelids, maxilla and eventually entering the lips [1]. Cleft number 10 of the Tessier classification is an extremely rare occurrence and is typically characterized by the presence of a cleft in the superior orbit along with a concomitant coloboma involving the middle one-third of the

Received: December 2, 2020 Final revision: April 1, 2021

Accepted: April 17, 2021 upper eyelid [2]. It may also present as a complete absence of eyelids or ablepharia. In certain cases, there will be an absence of the medial portion of the eyebrow, with the vertical angulation of lateral portion, often joining with the hairline of the scalp [3].

The ocular abnormalities which are associated mainly include congenital ocular surface problems like symblepharon, cutaneous pterygium and corneal abnormalities like corneal neovascularization and keratinization [4]. In few cases lower eyelid ectropion has also been reported.

Shao et al. [4] had published a classification and grading system of ocular involvement for Tessier cleft 10 patients, wherein the eyelid coloboma was graded from 0 to $3(0$, no upper eyelid defect; 1 , less than $1 / 3$ rd eyelid defect; 2 , greater than $1 / 3$ rd but less than $1 / 2$ eyelid defect; 3 , more than half eyelid defect); symblepharon and cutaneous pterygium were scored between 0 to 3 ( 0 , no symblepharon; 1 , symblepharon and cutaneous pterygium confined to the corneal limbus; 2, symblepharon and cutaneous pterygium extending up to the pupil margin; 3 , symblepharon and cu-
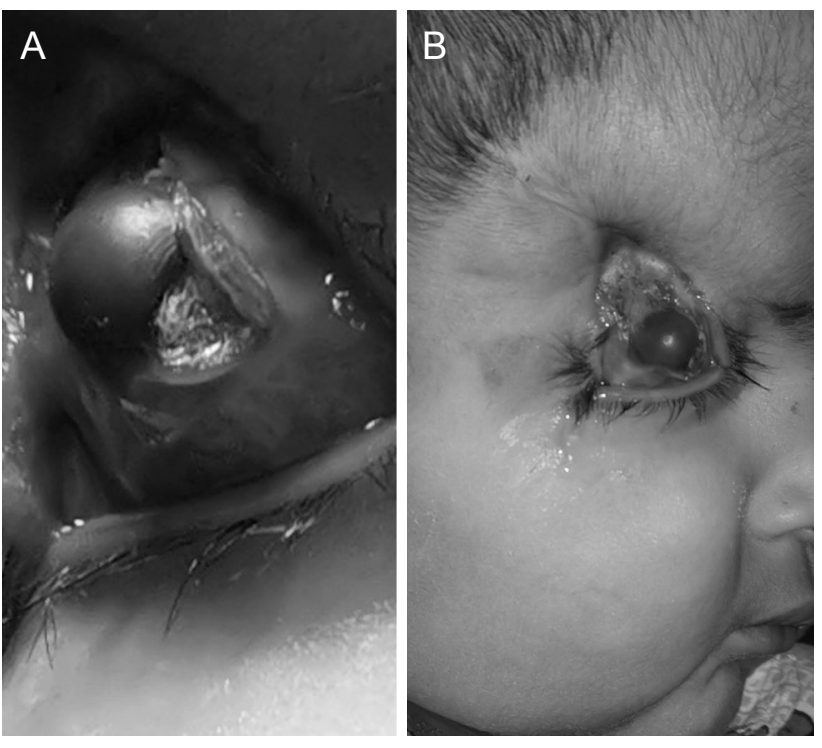

Fig. 1. (A) Right eye of the patient showing an anterior staphyloma with a disorganized globe. (B) Lateral view of the patient showing the upper eyelid with three parts; lateral and medial vertically oriented and the middle colobomatous part extending across the eyebrow into the forehead ending with a notch. Written informed consent for publication of the clinical images was obtained from the parents of the patient. 
taneous pterygium extending beyond the pupil into the central cornea); the severity of corneal involvement was graded from 0 to 3 ( 0 , clear cornea; 1 , corneal opacity and keratinization localized at the periphery less than two continuous hours; 2 , corneal opacity and cutaneous pterygium less than 4 clock hours; 3 , extensive opacity, cutaneous pterygium, keratinization, or neovascularization with more than four continuous hours); and lower eyelid ectropion was scored from 0 to 3 ( 0 , no ectropion appeared; 1 , less than one-third of the lower eyelid exhibited ectropion; 2 , greater than one-third but less than two-thirds of the eyelid exhibited ectropion; 3, more than two-thirds of the lower eyelid exhibit ectropion). Based on that our patient had a grade 3 upper eyelid defect with grade 3 corneal involvement with a grade 3 severe lower eyelid ectropion. Limb anomalies like syndactyly and cardiac defects like patent foramen oval are also known to be associated with cleft number 10, and thus it is recommended to conduct a thorough systemic evaluation including cardiac echo on encountering a similar patient [4].

The treatment of a Tessier number 10 cleft mainly involves various surgical techniques with the basic logic to provide corneal cover by reconstructing a cosmetically acceptable upper eyelid. Removal of the aberrant hairs can be accomplished using lasers, including a ruby laser, alexandrite laser, long pulsed Nd: YAG (neodymium-doped yttrium aluminum garnet) laser and intense pulse light treatment [2].

\section{Deepsekhar Das*, Aishwarya Rathod}

Oculoplasty, Ocular Oncology, and Pediatric Ophthalmology Services, Dr. Rajendra Prasad Centre for Ophthalmic Sciences, All India Institute of Medical Sciences, New Delhi, India

\section{Saloni Gupta*}

Department of Ophthalmology, Northern Railway Central Hospital, New Delhi, India

Sujeeth Modaboyina, Sahil Agrawal

Oculoplasty, Ocular Oncology, and Pediatric Ophthalmology Services, Dr. Rajendra Prasad Centre for Ophthalmic Sciences, All India Institute of Medical Sciences, New Delhi, India

E-mail (Sahil Agrawal): agrawalsahil03@aiims.edu

"These two authors contributed equally to this study.

\section{Conflict of Interest}

No potential conflict of interest relevant to this article was reported.

\section{References}

1. Tessier P. Anatomical classification facial, cranio-facial and latero-facial clefts. J Maxillofac Surg 1976;4:69-92.

2. Barnett JM, Pang JHY, Herman S, et al. Craniofacial cleft: a case of tessier 10 cleft with a novel method to repair large bilateral colobomas. Cleft Palate Craniofac J 2019;56:137780.

3. Lee HM, Noh TK, Yoo HW, et al. A wedge-shaped anterior hairline extension associated with a tessier number 10 cleft. Ann Dermatol 2012;24:464-7.

4. Shao C, Lu W, Li J, et al. Manifestation and grading of ocular involvement in patients with Tessier number 10 clefts. Eye (Lond) 2017;31:1140-5. 